\title{
Genes involved in tolerance to osmotic stress by random mutagenesis in Cronobacter malonaticus
}

\author{
Maofeng Zhang, ${ }^{*}{ }^{1}$ Xiyan Zhang, ${ }^{* 1}$ Liaowang Tong, ${ }^{* 1}$ Yaping Wang, ${ }^{*}$ Dexin Ou, ${ }^{*}$ Jumei Zhang, $\dagger$ \\ Qingping $\mathrm{Wu}, \mathrm{t}^{2}$ and Yingwang $\mathrm{Ye}^{*} \dagger^{2}$ \\ *School of Food Science and Engineering, Hefei University of Technology, Hefei 230009, China \\ †State Key Laboratory of Applied Microbiology Southern China, Guangdong Provincial Key Laboratory of Microbiology Culture Collection \\ and Application, Guangdong Institute of Microbiology, Guangzhou 510070, China
}

\section{ABSTRACT}

Cronobacter malonaticus is one of the opportunistic food-borne pathogens in powdered infant formula and has unusual abilities to survive under environmental stresses such as osmotic conditions. However, the genes involved in osmotic stress have received little attention in C. malonaticus. Here, genes involved in osmotic stress were determined in C. malonaticus using a transposon mutagenesis approach. According to the growth of mutants $(\mathrm{n}=215)$ under $5.0 \% \mathrm{NaCl}$ concentration, the survival of 5 mutants under osmotic stress was significantly decreased compared with that of the wild type strain. Five mutating sites, including potassium efflux protein KefA, inner membrane protein YqjF, peptidylprolyl isomerase, Cys-tRNA(Pro)/CystRNA(Cys) deacylase, and oligogalacturonate lyase were successfully identified. In addition, the biofilm formation of 5 mutants was determined using crystal violet staining, scanning electron microscopy, and confocal laser scanning microscopy, and the biofilms of 5 mutants significantly decreased within $72 \mathrm{~h}$ compared with that of wild type strain. This is the first report to determine the genes involved in osmotic tolerance in C. malonaticus. The findings provided valuable information for deep understanding of the mechanism of survival of C. malonaticus under osmotic stress, and a possible relationship between biofilm formation and tolerance to osmotic stress was also demonstrated in $C$. malonaticus.

Key words: Cronobacter malonaticus, random mutagenesis, osmotic stress, biofilm formation

\footnotetext{
Received October 15, 2017

Accepted December 28, 2017.

${ }^{1}$ These authors contributed equally to the manuscript.

${ }^{2}$ Corresponding authors: yeyw04@mails.gucas.ac.cn and wuqp203@163.com
}

\section{INTRODUCTION}

Cronobacter is a recently described genus, including Cronobacter sakazakii, Cronobacter malonaticus, Cronobacter turicensis, Cronobacter muytjensii, Cronobacter dublinensis, Cronobacter universalis, and Cronobacter condimenti (Caubilla-Barron et al., 2007). Cronobacter species are associated with necrotizing enterocolitis, bacteremia, and meningitis in infants, with mortality rates of up to 40 to $80 \%$ (Healy et al., 2010). Although Cronobacter has been isolated from food, soil, food processing environments, and clinical samples, powdered infant formula is considered to be the major transmission path of Cronobacter infections (Norberg et al., 2012; Ye et al., 2014).

Cronobacter has shown unusual abilities to survive under environmental stresses. Alvarez-Ordóñez et al. (2012) demonstrated that Cronobacter spp. showed stronger growth ability to survive under osmotic conditions than other Enterobacteriaceae. In addition, the heterogeneity about growth under osmotic stress among C. sakazakii strains was also observed. Transposon mutagenesis was used to reveal genes involved in osmotic stress and drying in C. sakazakii, and 29 genes were found to be response to osmotic stress (Alvarez-Ordonez et al., 2014). Riedel and Lehner (2007) identified differentially expressed proteins involved in osmotic stress using a comparative proteomics method. Genomic analysis showed that C. malonaticus is 1 of 3 Cronobacter species causing severe infections (Kucerova et al., 2010). Furthermore, the most reported cases indicated that C. malonaticus infected adults more than infants (Ogrodzki and Forsythe, 2017). Although only 1 case of meningitis from C. malonaticus has been documented in neonate, powdered infant formula is also an important point of origin for C. malonaticus (Hariri et al., 2013; Ogrodzki and Forsythe, 2017). Consequently, the potential risks of $C$. malonaticus to neonates should be great of concern. A high osmotic pressure encountered by Cronobacter during spray drying in the powdered formula manufacturing process affects its 
persistence in powdered infant formula (Amalaradjou and Venkitanarayanan, 2011). However, the factors involved in tolerance to osmotic stress in C. malonaticus have received little attention.

In our study, a transposon mutagenesis approach was used to construct a random mutagenesis library. Mutants showing significant growth differences compared with wild type strains (WT) in osmotic stress (5.0\% $\mathrm{NaCl}$ concentration) were screened, and the insertion sites were also identified by PCR amplification. Finally, the biofilm formation between mutants and WT were determined using crystal violet staining (CVS), scanning electron microscopy, and confocal laser scanning microscopy (CLSM).

\section{MATERIALS AND METHODS}

\section{Construction of a Random Mutagenesis Library}

We used a transposon mutagenesis approach to produce mutants. In brief, Escherichia coli WM3064 [pBSL180 with kanamycin (Km) and ampicillin (Amp) resistance] with deficiency of 2,6-diaminopimelic acid. Cronobacter malonaticus YE-001 identified using multilocus sequence typing (Baldwin et al., 2009) is sensitive to Km and Amp. Escherichia coli WM3064 and C. malonaticus YE-001 were used as the donor and receptor, respectively. The donor and receptor were inoculated into Luria Bertani (LB; Huankai, Guangzhou, China) broth at $37^{\circ} \mathrm{C}$ until reaching the exponential phase. The donor and receptor $(3: 1)$ were then mixed and doped onto filter membrane at $37^{\circ} \mathrm{C}$ for incubation of $20 \mathrm{~h}$. Finally, the filter membrane was washed using sterile LB broth, and streaked onto chromogenic Enterobacter sakazakii agar (kanamycin) and chromogenic Enterobacter sakazakii agar (ampicillin kanamycin) at $37^{\circ} \mathrm{C}$ for $12 \mathrm{~h}$. The true mutants can grow on chromogenic Enterobacter sakazakii agar (kanamycin) and cannot grow on chromogenic Enterobacter sakazakii agar (ampicillin).

\section{Screening of Mutants of Significant Differences Under Osmotic Stress}

For testing bacterial growth under osmotic conditions, overnight culture (optical density at $600 \mathrm{~nm}=$ $0.8, \mathrm{vol} / \mathrm{vol}, 1 \%$ ) was inoculated into LB broth containing $5.0 \% \mathrm{NaCl}$ at $37^{\circ} \mathrm{C}$ for $8 \mathrm{~h}$. Growth of mutants (n $=215$ ) and WT were measured spectrophotometrically in 96-well culture plates by determining the optical density at $600 \mathrm{~nm}$. Mutants having significant growth differences compared with WT were subject to further study.

\section{Mapping of Transposon Insertion Sites}

The transposon insertion sites were identified by PCR described by Chen et al. (2010) and Merrell et al. (2002) with modification. In brief, chromosomal DNA was extracted using Bacteria DNA Exaction Kit (Tiangen, Beijing, China). The forward primer (5'-GGCCACGCGTCGACTAGTCAGACTACGGCTAGCTG-3') and the reverse primer (5'-CATCCGGGGTCAGCACCGTTTC-3') were used. The first PCR amplification was performed for $5 \mathrm{~min}$ at $94^{\circ} \mathrm{C}$; followed by 6 cycles of $94^{\circ} \mathrm{C}$ for $30 \mathrm{~s}, 30^{\circ} \mathrm{C}$ for $30 \mathrm{~s}$, $72^{\circ} \mathrm{C}$ for $90 \mathrm{~s}$; then 30 cycles of $94^{\circ} \mathrm{C}$ for $30 \mathrm{~s}, 45^{\circ} \mathrm{C}$ for $30 \mathrm{~s}, 72^{\circ} \mathrm{C}$ for $2 \mathrm{~min}$; and finally $10 \mathrm{~min}$ at $72^{\circ} \mathrm{C}$. The PCR products were purified for the second PCR amplification, the forward primer was 5'-GGCCACGTCGACTAGTCA-3', whereas the reverse primer was 5'-GGATCCACTAGTTCTAGAGCGGC-3'. The thermal conditions for the second amplification were 5 min at $94^{\circ} \mathrm{C} ; 30$ cycles of $94^{\circ} \mathrm{C}$ for $30 \mathrm{~s}, 52^{\circ} \mathrm{C}$ for $30 \mathrm{~s}$, $72^{\circ} \mathrm{C}$ for $2 \mathrm{~min}$; and $10 \mathrm{~min}$ at $72^{\circ} \mathrm{C}$. The second PCR products were purified for being sequenced using as a primer (5'-GGATCCACTAGTTCTAGAGCGGC-3'). Insertion sites or mutating sites were identified by sequences alignment on NCBI.

\section{Quantitative Analysis of Biofilm Formation}

For CVS detection, C. malonaticus strains were inoculated into $\mathrm{LB}$ containing $5.0 \% \mathrm{NaCl}$ for incubation at $37^{\circ} \mathrm{C}$ for $8 \mathrm{~h}$ with constant shaking. Twenty microliters of overnight culture (optical density at $590 \mathrm{~nm}=0.5$ ) were inoculated into 48 -well polystyrene plates containing $1.98 \mathrm{~mL}$ of sterile LB broth and incubated at $37^{\circ} \mathrm{C}$ for 24,48 , and $72 \mathrm{~h}$. The plates were rinsed 3 times with deionized water and the adherent bacteria cells were stained with $1 \%$ crystal violet for $30 \mathrm{~min}$. After being rinsed 3 times with deionized water, the crystal violet was liberated by $30 \%$ acetic acid following a 10-min incubation. The optical density values of biofilm in each well were measured at $590 \mathrm{~nm}$ and each experiment was done in triplicate. The statistics analysis about biofilm-forming abilities among mutants and WT using Originpro 8.5.1 (OriginLab Corp., Northampton, MA).

For scanning electron microscopy, glass coverslips (Jingan, Shanghai, China) were immersed in tryptic soy broth and $20 \mu \mathrm{L}$ of $C$. malonaticus (WT and 5 mutants) were transferred to 48-well plates (Baiyan, Shanghai, China) containing $1.98 \mathrm{~mL}$ of LB with $5.0 \%$ $\mathrm{NaCl}$ for incubation at $37^{\circ} \mathrm{C}$ for 24,48 , and $72 \mathrm{~h}$ respectively. During this biofilm-formation period, the glass coverslips from different incubation times were prepared and detected by scanning electron microscopy (Hitachi, 
Tokyo, Japan) as described previously by Wang et al. (2013). For CLSM, the glass coverslips were prepared the same as scanning electron microscopy detection, and bacterial biofilm formation on glass slips was stained with LIVE/DEAD BacLight bacterial viability Kit according to instructions (Invitrogen, Carlsbad, $\mathrm{CA}$ ), and were subsequently observed by CLSM (Zeiss, Berlin, Germany).

\section{RESULTS AND DISCUSSION}

Cronobacter spp. is an important food-borne pathogens associated with severe but rare infections in neonates. Cronobacter malonaticus predominates in adult infection cases, and the contaminated level of C. malonaticus in powdered infant formula is also high (Hariri et al., 2013; Ogrodzki and Forsythe, 2017). From our mutant library, 5 mutants were successfully screened, and the growth of 5 mutants under osmotic stress was significantly $(P<0.01)$ decreased compared with those of WT, as shown in Figure 1. Furthermore, using arbitrary PCR detection, the transposon insertion sites were successfully identified and shown in Table 1. These mutating genes encode inner membrane protein YqjF, potassium efflux protein KefA, Peptidylprolyl isomerase (PPIase), Cys-tRNA(Pro)/Cys-tRNA(Cys) deacylase, and oligogalacturonate lyase. Cronobacter spp. has unusual abilities to survival under different environmental stresses. A total of 29 genes in $C$. sakazakii were involved in osmotic stress belonging to various clusters of orthologous groups functional categories by using a transposon mutagenesis method (Alvarez-Ordonez et al., 2014). In another paper, proteins upregulated in response to osmotic stress fell into DNA/RNA stabilizing enzymes, molecular chaperones,

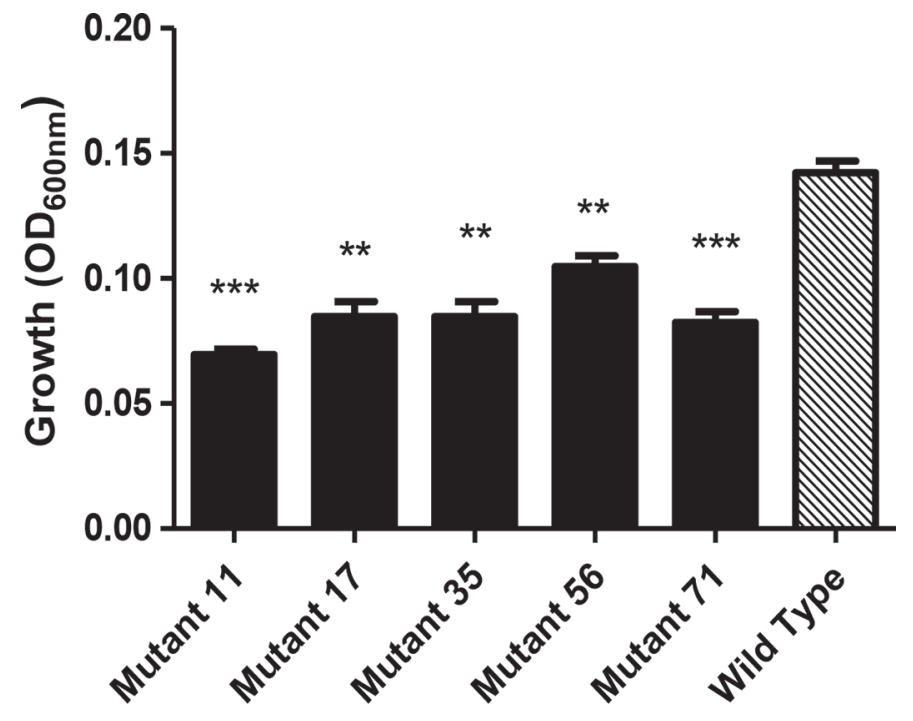

Figure 1. Cronobacter malonaticus growth of mutants and wild type strains in Luria Bertani broth with $5 \% \mathrm{NaCl} . \mathrm{OD}_{600 \mathrm{~mm}}=$ optical density at $600 \mathrm{~nm}$. Error bars represent SD. ${ }^{* *} P<0.01,{ }^{* * *} P<0.001$.

and oxidative-related proteins through the comparative proteomics analysis (Riedel and Lehner, 2007).

The inner membrane protein $\mathrm{YqjF}$ is a conserved inner membrane protein of unknown function. In our study, the deletion of $Y q j F$ caused impaired growth rates under osmotic stress in $C$. malonaticus. Functions of other inner membrane proteins were preliminarily studied previously (Baba et al., 2006; Mamat et al., 2008; Anderson et al., 2017). Baba et al. (2006) stated that $y h j D$ is a nonessential gene in WT E. coli. In another study, single AA substitutions in $y$ hjD could confer viability to 3-deoxy-D-manno-oct-2-ulosic aciddepleted E. coli (Mamat et al., 2008). In E. coli, the

Table 1. Mapping of transposon insertion sites in Cronobacter malonaticus YE01

\begin{tabular}{|c|c|c|c|}
\hline Mutant $^{1}$ & Gene encoded proteins (organism) & Relevant features & Locus tag \\
\hline Mutant 17 & $\begin{array}{l}\text { Potassium efflux protein KefA (Cronobacter } \\
\text { malonaticus CMCC } 45402 \text { ) }\end{array}$ & Transport potassium & P262_04184 ${ }^{3}$ \\
\hline Mutant 35 & $\begin{array}{l}\text { Peptidylprolyl isomerase (Cronobacter malonaticus } \\
\text { CMCC } 45402 \text { ) }\end{array}$ & $\begin{array}{l}\text { Catalyze the isomerization of peptide } \\
\text { bonds N-terminal of prolines }\end{array}$ & P262_04121 $1^{4}$ \\
\hline Mutant 56 & $\begin{array}{l}\text { Cys-tRNA(pro)/cys-tRNA(cys) deacylase (Cronobacter } \\
\text { malonaticus CMCC } 45402 \text { ) }\end{array}$ & $\begin{array}{l}\text { Can hydrolyze misacylated cys-tRNA(pro) } \\
\text { and cys-tRNA(cys) }\end{array}$ & P262_04972 \\
\hline
\end{tabular}

\footnotetext{
${ }^{1}$ Mutants all from the current study.

${ }^{2}$ https://www.ncbi.nlm.nih.gov/protein/564116232.

${ }^{3}$ https://www.ncbi.nlm.nih.gov/protein/AHB71343.1.

${ }^{4}$ https://www.ncbi.nlm.nih.gov/protein/564116355.

${ }^{5}$ https://www.ncbi.nlm.nih.gov/protein/AHB71899.1.

${ }^{6}$ https://www.ncbi.nlm.nih.gov/protein/564116801.
} 
synthesis of inner membrane protein YhiM is necessary for growth at high temperatures and low osmolarity (Anderson et al., 2017).

Potassium efflux protein KefA has received little attention on its detailed function. Other potassium efflux proteins, such as bacterial KefC protein, were originally considered to be the $\mathrm{K}^{+}$channel (Booth et al., 2003). Another study indicated that KefC functioned as a $\mathrm{K}^{+}$/ $\mathrm{H}^{+}$antiporter mode of action (Fujisawa et al., 2007). A previous study showed that increasing acute hyperosmotic $\mathrm{NaCl}$ stress causes a dose-dependent $\mathrm{K}^{+}$leak from the cell, resulting in a substantial decrease in cytosolic $\mathrm{K}+$ content and a concurrent accumulation of $\mathrm{Na}^{+}$ in the cell (Shabala et al., 2009; Roosild et al., 2009; Aranda-Sicilia et al., 2012). In our study, the deletion of potassium transporter protein KefA caused decreasing survival of $C$. malonaticus under osmotic stress, which might result from loss of $\mathrm{K}^{+}$channel under high $\mathrm{NaCl}$ concentrations and interruption of intracellular and extracellular ion balance.

The function of PPIase is to catalyze the isomerization of peptide bonds N-terminal of prolines, and PPIase plays an important role in protein folding or trafficking (Schmidpeter et al., 2015; Kang et al., 2017; Liu et al., 2017). The PPIase may be involved in the salt adaptation of Shewanella sp. WP3 (Li et al., 2006). In the present study, PPIase also contributed to survival of C. malonaticus under osmotic stress. We inferred that the structure or functions of some proteins may be interfered due to the absence of PPIase gene in mutant strain, which significantly affected the metabolic procession and growth under osmotic stress.

The Cys-tRNA(Pro)/Cys-tRNA(Cys) deacylase can hydrolyze misacylated Cys-tRNA(Pro) and CystRNA(Cys). Previous reports showed that the hydrolysis of aminoacyl-tRNA could influence cell growth (Ruan and Soll, 2005; Liu et al., 2015). In our study, Cys-tRNA(Pro)/Cys-tRNA(Cys) deacylase significantly reduced the growth of $C$. malonaticus under osmotic stress. However, information about effects of the gene on the survival in other food-borne pathogens under osmotic stress is not available.

Oligogalacturonate lyase (OGL) is an enzyme of pectate lyases in Enterobacteriaceae; OGL in Yersinia enterocolitica use a $\beta$-elimination mechanism to preferentially catalyze the conversion of saturated and unsaturated digalacturonate into monogalacturonate (Abbott et al., 2010). The present study showed that the

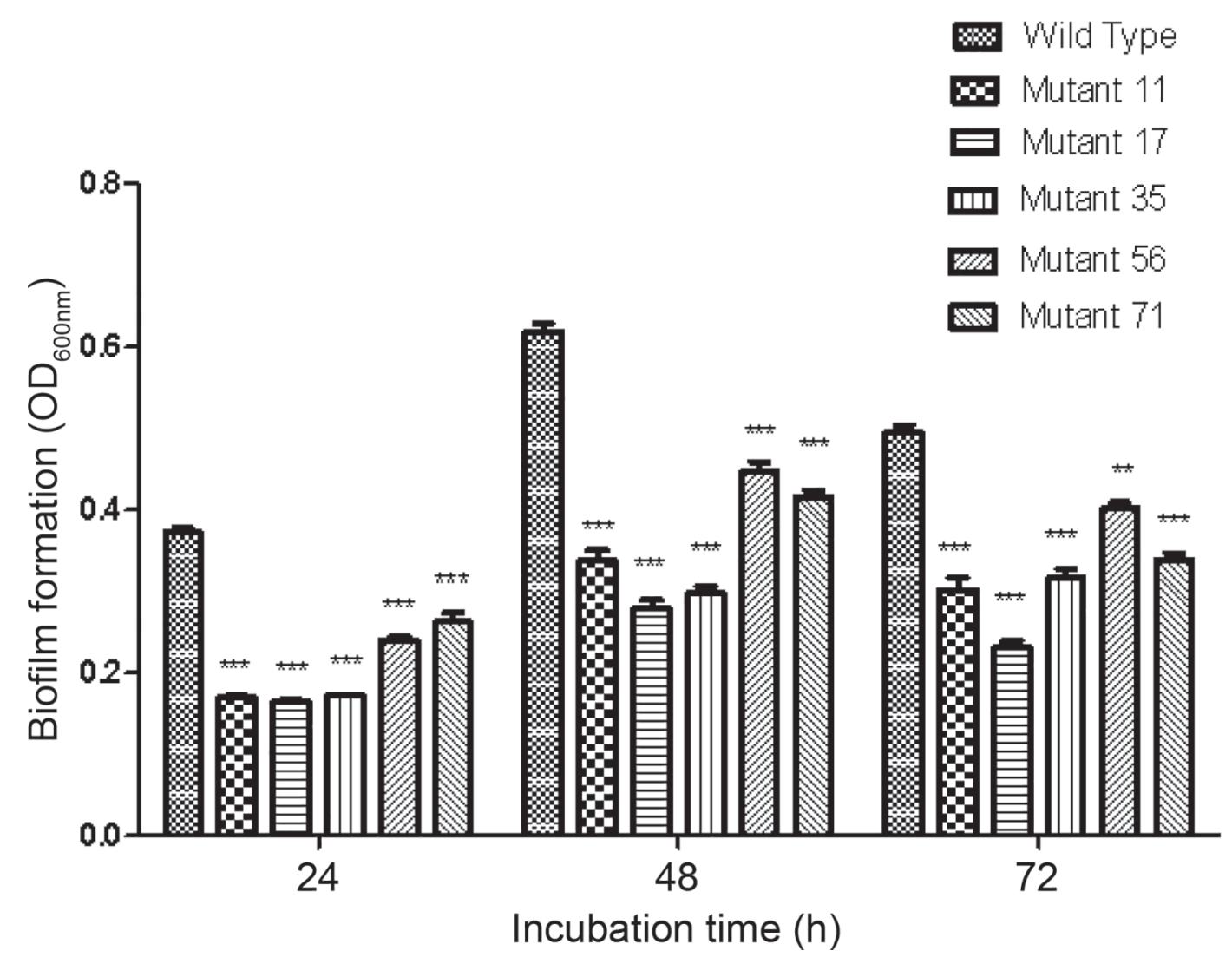

Figure 2. Biofilm formation of Cronobacter malonaticus mutants and wild type strains in Luria Bertani broth with $5 \% \mathrm{NaCl}$ using crystal violet staining. $\mathrm{OD}_{600 \mathrm{~nm}}=$ optical density at $600 \mathrm{~nm}$. Error bars represent $\mathrm{SD}$. ${ }^{* *} P<0.01,{ }^{* * *} P<0.001$. 
$24 \mathrm{~h}$

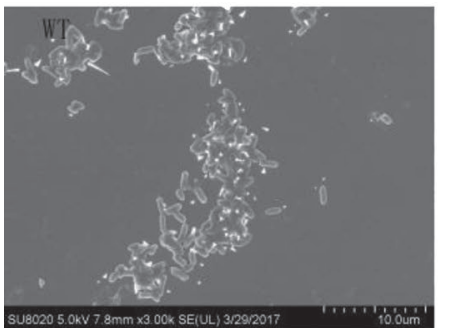

Mutant 11 s:
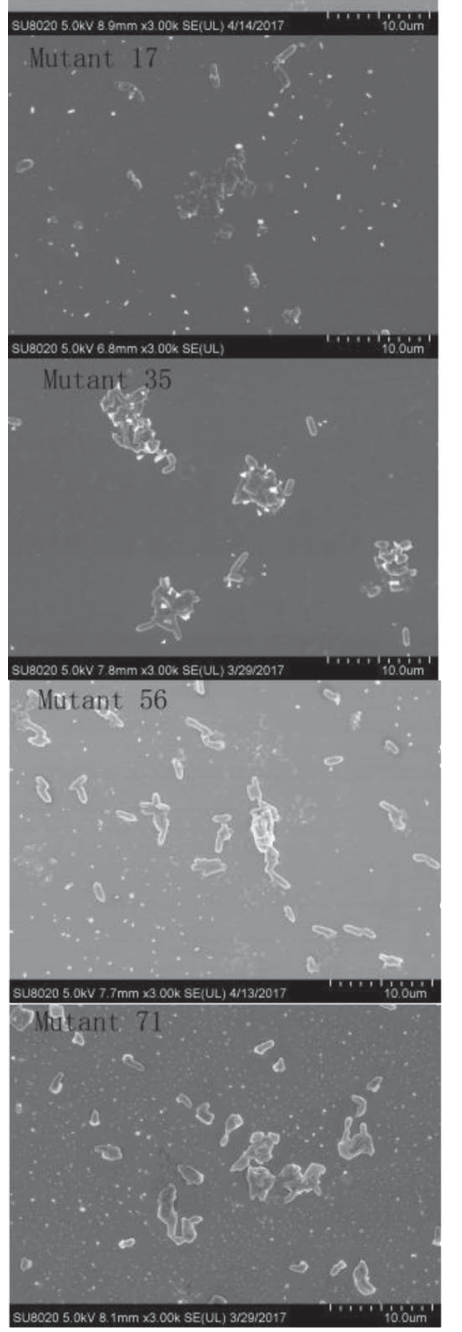

$48 \mathrm{~h}$
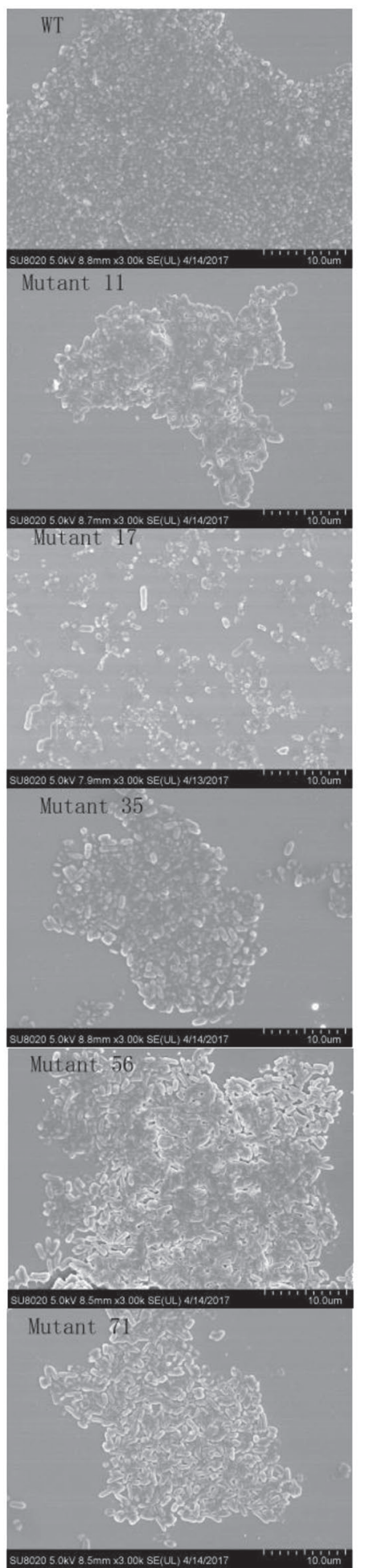

$72 \mathrm{~h}$
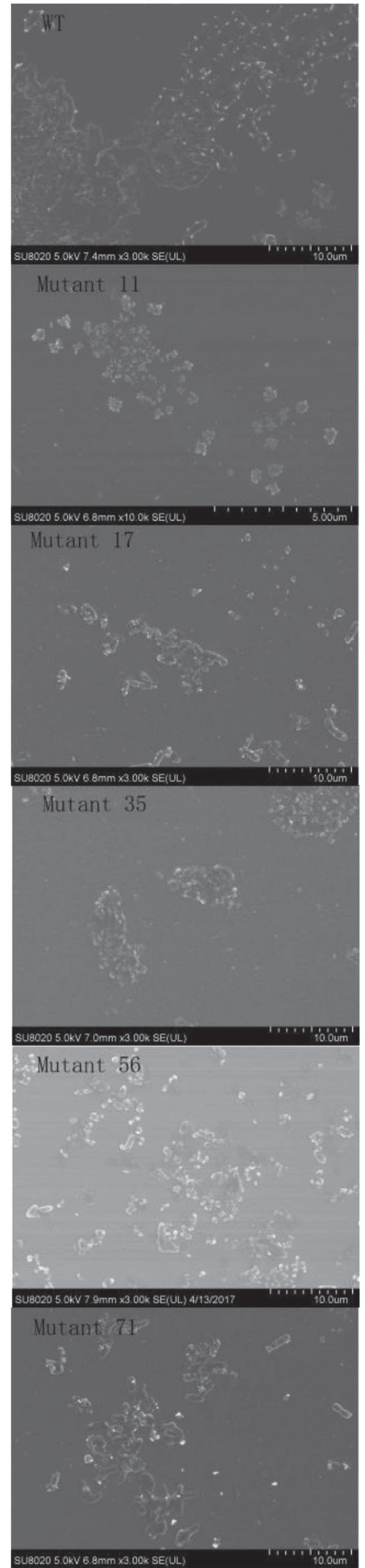

Figure 3. Biofilm formation of Cronobacter malonaticus mutants and wild type strains (WT) in Luria Bertani broth with $5 \% \mathrm{NaCl}$ using scanning electron microscopy. 


\section{$24 \mathrm{~h}$}
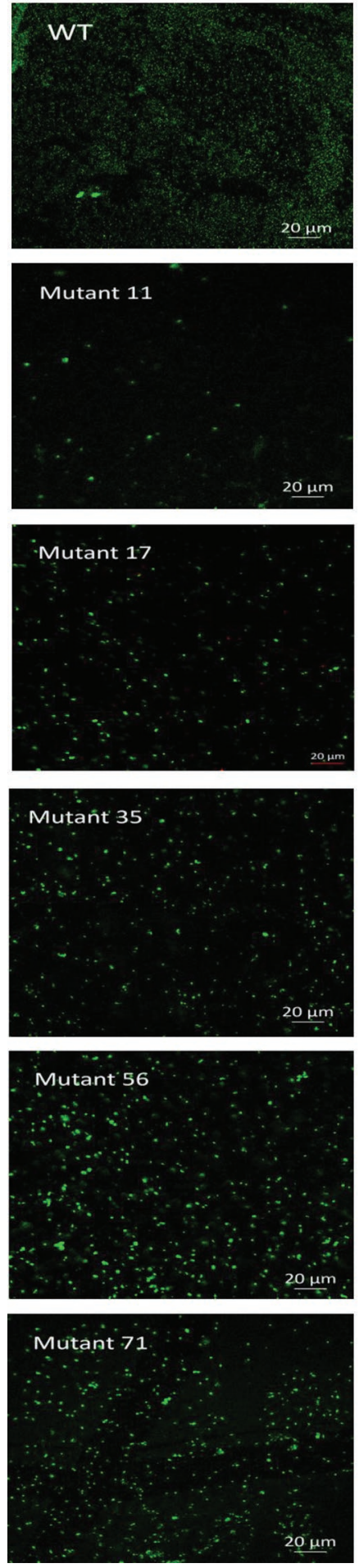

$48 \mathrm{~h}$
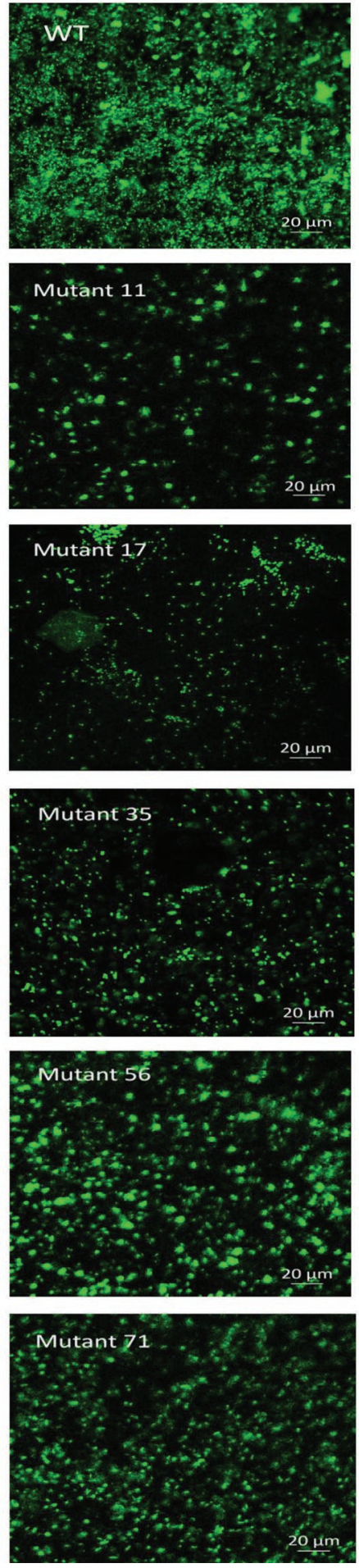

$72 \mathrm{~h}$

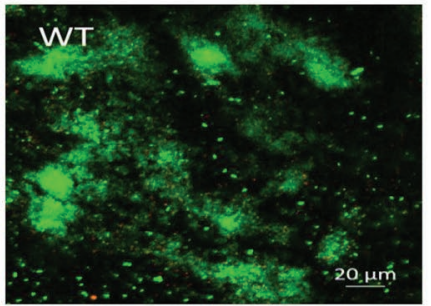

Mutant 11
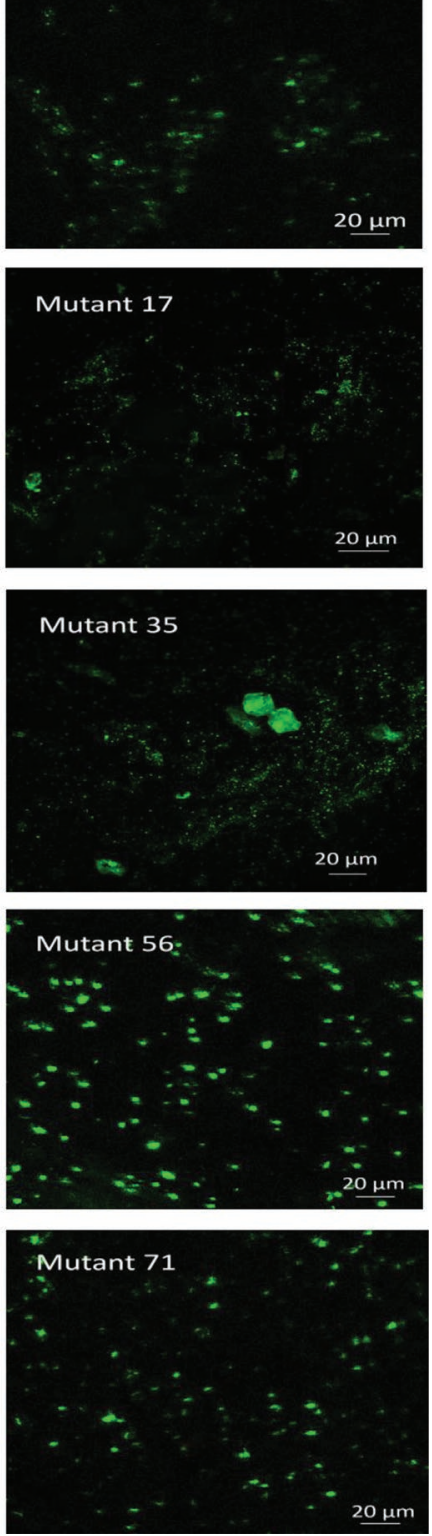

Figure 4. Biofilm-forming ability of Cronobacter malonaticus mutants and wild type strains (WT) in Luria Bertani broth with $5 \% \mathrm{NaCl}$ using confocal laser scanning microscopy. Color version available online. 
deletion of OGL gene had negative effects on survival of C. malonaticus; however, the detailed mechanism of OGL on survival in C. malonaticus remains to be revealed.

Biofilms are an interface-associated consortia of microorganisms that are typically embedded in an endogenous slimy matrix referred to as extracellular polymeric substance, which enhance resistance to a variety of environmental stresses (Hartmann et al., 2010). Furthermore, the biofilm formation of 5 mutants and WT was determined using CVS, scanning electron microscopy, and CLSM. The mutants and WT formed the highest number of biofilms after $48 \mathrm{~h}$ of incubation, as shown in Figure 2. Different biofilm-forming abilities were observed among C. sakazakii strains under different conditions (Ye et al., 2016). In addition, we also found that the biofilm-forming abilities in mutants significantly decreased compared with those of WT (Figures 3 and 4). The 5 mutant strains formed weaker biofilms than WT within $72 \mathrm{~h}$ of incubation under osmotic stress. In recent years, the biofilm formation in Cronobacter spp. under different environmental conditions has been described (Lee et al., 2012; Jung et al., 2013; Ye et al., 2015). Hartmann et al. (2010) demonstrated that cellulose biosynthesis, flagellar structure and biosynthesis, and basic cellular procession were involved in biofilm formation in C. sakazakii. Furthermore, k-antigen and colonic acid capsular profiles among $C$. sakazakii and $C$. malonaticus were determined (Ogrodzki and Forsythe, 2015), which showed that capsular type [K2:CA2:Cell $\left.{ }^{+}\right]$ were associated with neonatal meningitis and necrotizing enterocolitis. They also found that K2:CA2:Cell ${ }^{+}$ was found in C.turicensis, C. dublinensis, and C. universalis (Ogrodzki and Forsythe, 2017).

We found that the deletion of inner membrane protein YqjF, potassium efflux protein KefA, PPIase, Cys-tRNA(Pro)/Cys-tRNA(Cys) deacylase, and OGL caused the significant reduction of biofilm formation in C. malonaticus. Ye et al. (2016) found that PPIase might be the potential protein contributing to biofilm formation in C. sakazakii using a comparative proteomics method. In addition, the lowest amount of biofilm formation in KefA mutant strain (mutant 17) was observed among 5 mutants. In Bacillus subtilis, the expression of mstX and the downstream putative $\mathrm{K}^{+}$ efflux channel, yugO, is necessary for biofilm formation (Lundberg et al., 2013). Biofilm formation is a very complex processing associated with various factors. Other factors including inner membrane protein YqjD, potassium efflux protein KefA, Cys-tRNA(Pro)/ Cys-tRNA(Cys) deacylase, and OGL were found to be associated with biofilm formation in C. malonaticus. In addition, the findings also confirmed a positive relationship between survival and biofilm-forming abili- ties under osmotic stresses in C. malonaticus, but the detailed mechanism of these genes on biofilm formation remains to be revealed in the future.

\section{ACKNOWLEDGMENTS}

We gratefully acknowledge M. Y. Xu (Guangdong Institute of Microbiology) for presenting the E. coli WM3064 and the financial support of the National Key Research and Development program (2017YFC1601200), National Natural Science Foundation of China (31671951), the Anhui provincial Grand Project special of Science and Technology (15czz03109), the Science and Technology Planning Project of Guangdong Province (Guangzhou, China; 2016A050502033), Project of Science and Technology in Guangzhou (China; 201604020036), and State Key Laboratory of Applied Microbiology Southern China Open Foundations, Guangzhou, China (SKLAM004-2015).

\section{REFERENCES}

Abbott, D. W., H. J. Gilbert, and A. B. Boraston. 2010. The active site of oligogalacturonate lyase provides unique insights into cytoplasmic oligogalacturonate $\beta$-Elimination. J. Biol. Chem. 285:39029-39038.

Alvarez-Ordonez, A., M. Begley, T. Clifford, T. Deasy, B. Collins, and C. Hill. 2014. Transposon mutagenesis reveals genes involved in osmotic stress and drying in Cronobacter sakazakii. Food Res. Int. 55:45-54.

Alvarez-Ordóñez, A., M. Begley, and C. Hill. 2012. Polymorphisms in rpoS and stress tolerance heterogeneity in natural isolates of Cronobacter sakazakii. Appl. Environ. Microbiol. 78:3975-3984.

Amalaradjou, M. A. R., and K. Venkitanarayanan. 2011. Effect of trans-cinnamaldehyde on reducing resistance to environmental stresses in Cronobacter sakazakii. Foodborne Pathog. Dis. 8:403409.

Anderson, M. A., M. D. Mann, M. A. Evans, and R. L. Sparks-Thissen. 2017. The inner membrane protein YhiM is necessary for Escherichia coli growth at high temperatures and low osmolarity. Arch. Microbiol. 199:171-175.

Aranda-Sicilia, M. N., O. Cagnac, S. Chanroj, H. Sze, M. P. RodríguezRosales, and K. K. Venema. 2012. Arabidopsis KEA2, a homolog of bacterial KefC, encodes a $\mathrm{K}^{+} / \mathrm{H}^{+}$antiporter with a chloroplast transit peptide. Biochim. Biophys. Acta 1818:2362-2371.

Baba, T., T. Ara, M. Hasegawa, Y. Takai, Y. Okumura, and M. Baba. 2006. Construction of Escherichia coli K-12 in-frame, single-gene knockout mutants: the Keio collection. Mol. Syst. Biol. 2:0008.

Baldwin, A., M. Loughlin, J. Caubilla-Barron, E. Kucerova, G. Manning, C. Dowson, and S. Forsythe. 2009. Multilocus sequence typing of Cronobacter sakazakii and Cronobacter malonaticus reveals stable clonal structures with clinical significance which do not correlate with biotypes. BMC Microbiol. 9:223.

Booth, I. R., M. D. Edwards, and S. Miller. 2003. Bacterial ion channels. Biochemistry 42:10045-10053.

Caubilla-Barron, J., E. Hurrell, S. Townsend, P. Cheetham, C. LocCarrillo, O. Fayet, M. F. Prere, and S. J. Forsythe. 2007. Genotypic and phenotypic analysis of Enterobacter sakazakii strains from an outbreak resulting in fatalities in a neonatal intensive care unit in France. J. Clin. Microbiol. 45:3979-3985.

Chen, X., M. Y. Xu, J. B. Wei, and G. P. Sun. 2010. Two different electron transfer pathoway may involve in azoreduction by Shewanella decolorationis S12. Appl. Microbiol. Biotechnol. 86:743-751. 
Fujisawa, M., M. Ito, and T. A. Krulwich. 2007. Three two-component transporters with channel-like properties have monovalent cation/ proton antiport activity. Proc. Natl. Acad. Sci. USA 104:1328913294.

Hariri, S., S. Joseph, and S. J. Forsythe. 2013. Cronobacter sakazakii ST4 strains and neonatal meningitis, US. Emerg. Infect. Dis. 19:175-177.

Hartmann, I., P. Carranza, A. Lehner, R. Stephan, L. Eberl, and K. Riedel. 2010. Genes Involved in Cronobacter sakazakii biofilm formation. Appl. Environ. Microbiol. 76:2251-2261.

Healy, B., S. Cooney, S. O. Brien, C. Iversen, P. Whyte, J. Nally, J. J. Callanan, and S. Fanning. 2010. Cronobacter (Enterobacter sakazakii): An opportunistic foodborne pathogen. Foodborne Pathog. Dis. 7:339-350.

Jung, J. H., N. Y. Choi, and S. Y. Lee. 2013. Biofilm formation and exopolysaccharide (EPS) production by Cronobacter sakazakii depending on environmental conditions. Food Microbiol. 34:70-80.

Kang, D. S., S. Y. Moon, H. J. Cho, J. M. Lee, and I. S. Kong. 2017. Interaction of a $22 \mathrm{kDa}$ peptidyl prolyl cis/trans isomerase with the heat shock protein DnaK in vibrio anguillarum. J. Microbiol. Biotechnol. 27:644-647.

Kucerova, E., S. W. Clifon, X. Q. Xia, F. Long, S. Porwollik, L. Fulton, C. Fronick, P. Minx, K. Kyung, W. Warren, R. Fulton, D. Feng, A. Wollam, N. Shah, V. Bhonagiri, W. E. Nash, K. Hallwworth-Pepin, R. K. Wilson, M. McClelland, and S. J. Forsythe. 2010. Genome sequence of Cronobacter sakazakii BAA-894 and comparative genomic hybridization analysis with other Cronobacter species. PLoS One 5:e9556.

Lee, Y.-D., J.-H. Park, and H. Chang. 2012. Detection, antibiotic susceptibility and biofilm formation of Cronobacter spp. from various foods in Korea. Food Control 24:225-230.

Li, S., X. Xiao, J. Y. Li, J. X. Luo, and F. W. Wang. 2006. Identification of genes regulated by changing salinity in the deep-sea bacterium Shewanella sp. WP3 using RNA arbitrarily primed PCR. Extremophiles 10:97-104.

Liu, S., C. L. Guo, Z. Dang, and X. J. Liang. 2017. Comparative proteomics reveal the mechanism of Tween 80 enhanced phenanthrene biodegradation by Sphingomonas sp. GY2B. Ecotoxicol. Environ. Saf. 137:256-264.

Liu, Z., V. R. Oscar, G. Yuki, M. N. Eva, R. P. Lluis, S. Hiroaki, and M. F. Karin. 2015. Homologous trans-editing factors with broad tRNA specificity prevent mistranslation caused by serine/threonine misactivation. Proc. Natl. Acad. Sci. USA 112:6027-6032.

Lundberg, M. E., E. C. Becker, and S. Choe. 2013. MstX and a putative potassium channel facilitate biofilm formation in Bacillus subtilis. PLoS One 8:e60993.

Mamat, U., T. C. Meredith, P. Aggarwal, A. Kuhl, P. Kirchhoff, B. Lindner, A. Hanuszkiewicz, J. Sun, O. Holst, and R. W. Woodard.
2008. Single amino acid substitutions in either YhjD or MsbA confer viability to 3-deoxy-D-manno-oct-2-ulosonic acid-depleted Escherichia coli. Mol. Microbiol. 67:633-648.

Merrell, D. S., D. L. Hava, and A. Camilli. 2002. Identification of novel factors involved in colonization and acid tolerance of Vibrio cholerae. Mol. Microbiol. 43:1471-1491.

Norberg, S., C. Stanton, R. P. Ross, C. Hill, G. F. Fitzgerald, and P. D. Cotter. 2012. Cronobacter spp. in powdered infant formula. J. Food Prot. 75:607-620.

Ogrodzki, P., and S. J. Forsythe. 2015. Capsular profiling of the Cronobacter genus and the association of specific Cronobacter sakazakii and $C$. malonaticus capsule types with neonatal meningitis and necrotizing enterocolitis. BMC Genomics 16:758.

Ogrodzki, P., and S. J. Forsythe. 2017. DNA-sequence based typing of the Cronobacter genus using MLST, CRISPR-cas array and capsular profiling. Front. Microbiol. 8:1875.

Riedel, K., and A. Lehner. 2007. Identification of proteins involved in osmotic stress response in Enterobacter sakazakii by proteomics. Proteomics 7:1217-1231.

Roosild, T. P. S. Castronovo, S. Miller, C. Li, T. Rasmussen, W. Bartlett, B. Gunasekera, S. Choe, and I. R. Booth. 2009. KTN (RCK) domains regulate $\mathrm{K}^{+}$channels and transporters by controlling the dimer-hinge conformation. Structure 17:893-903.

Ruan, B., and D. Soll. 2005. The bacterial YbaK protein is a CystRNA Pro and Cys-tRNA Cys deacylase. J. Biol. Chem. 280:25887-25891.

Schmidpeter, P. A. M., J. R. Koch, and F. X. Schmid. 2015. Control of protein function by prolyl isomerization. Biochim. Biophys. Acta 1850:1973-1982.

Shabala, L., J. Bowman, J. Brown, T. Ross, T. McMeekin, and S. Shabala. 2009. Ion transport and osmotic adjustment in Escherichia coli in response to ionic and non-ionic osmotica. Environ. Microbiol. 11:137-148.

Wang, C. Y., C. P. Hsu, H. W. Huang, and B. B. Yang. 2013. The relationship between inactivation and morphological damage of Salmonella enterica treated by high hydrostatic pressure. Food Res. Int. 54:1482-1487.

Ye, Y. W., R. Jiao, J. N. Gao, H. Li, N. Ling, Q. P. Wu, J. M. Zhang, and X. K. Xu. 2016. Proteins involved in responses to biofilm and planktonic modes in Cronobacter sakazakii. Lebensm. Wiss. Technol. 65:1093-1099.

Ye, Y. W., H. Li, Q. P. Wu, J. M. Zhang, and Y. D. Lu. 2014. The Cronobacter sp. in milk and dairy products: Detection and typing. Int. J. Dairy Technol. 67:167-175.

Ye, Y. W., N. Ling, R. Jiao, Q. P. Wu, Y. J. Han, and J. N. Gao. 2015. Effects of culture conditions on the biofilm formation of Cronobacter sakazakii strains and distribution of genes involved in biofilm formation. Lebensm. Wiss. Technol. 61:1-6. 